\title{
Global stability of coupled Markovian switching reaction-diffusion systems on networks
}

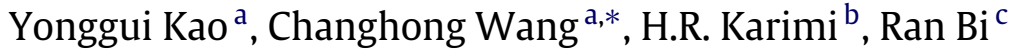 \\ ${ }^{a}$ Control and Inertial Technology Research Center, Harbin Institute of Technology, Harbin, Heilongjiang Province, 150001, PR China \\ ${ }^{\mathrm{b}}$ Department of Engineering, Faculty of Technology and Science, University of Agder, N-4898 Grimstad, Norway \\ ' School of Mechanical Engineering and Automation, Harbin Institute of Technology, Shenzhen University Town, Xili, Nanshan District, \\ Shenzhen, PR China
}

Received 18 July 2012

Accepted 27 December 2013

\section{Introduction}

Coupled systems on networks (CSNs) are composed of a large number of highly interconnected dynamical nodes. Each node is a unit with specific contents [1]. In the real world, many systems are modeled as coupled systems on networks (CSNs), such as communication networks, social networks, power grids, cellular networks, World Wide Web, metabolic systems, food webs, disease transmission networks, etc. [2,3]. When studying complex networks' dynamics, one problem is learning how a large ensemble of dynamical systems can behave collectively [4]. Therefore, it is very necessary to construct a relation between the stability criteria of a CSNs and some topology property of the network [5-7]. Li and Shuai [8] have considered global stability for the general CSNs based on graph theory, without discussing the stochastic effects.

Many large-scale dynamical systems from science and engineering often are represented as stochastic coupled systems on networks (SCSNs) [9-11], which could be described in a directed graph. Kao, Sun and Cao [12] have considered stability of coupled stochastic systems with time delay on networks without reaction-diffusion effects. However, for many realistic networks, the node state in CSNs is seriously dependent on the time and space. Hence, in order to describe more accurately the dynamic changes of CSNs, reaction-diffusion effects should also be considered [13-18]. Kao and Wang have considered global stability analysis for stochastic coupled reaction-diffusion systems on networks [19]. Markovian jump systems,

is This work is supported by the National Natural Science Foundations of China $(60973048,60974025,60673101,60939003)$, National 863 Plan Project (2008AA04Z401, 2009AA043404), the Natural Science Foundation of Shandong Province (No. Y 2007G30), the Scientific and Technological Project of Shandong Province (No. 2007GG3WZ04016), the Natural Scientific Research Innovation Foundation in Harbin Institute of Technology (HIT.NSRIF.2001120), the China Postdoctoral Science Foundation (20100481000) and the Shandong Provincial Key Laboratory of Industrial Control Technique (Qingdao University).

* Corresponding author.

E-mail addresses: ygkao2008@gmail.com (Y. Kao),chwang@hit.edu.cn (C. Wang), hamid.r.karimi@uia.no (H.R. Karimi). 
introduced by Krasovskii and Lidskii [20] in 1961, have received increasing attentions [21-38], because the hybrid systems driven by continuous-time Markov chains have been used to model many practical systems such as components failures or repairs, changing subsystem interconnections, and abrupt environmental disturbances, please see [22] and the references therein. The CSNs may be driven by continuous-time Markov chains. To the best of the authors' knowledge, stability analysis for Markovian switching reaction-diffusion coupled systems on networks (MSRDCSNs) has not been properly addressed, which still remains important and challenging.

Motivated by the above discussions, in this paper, we propose the MSRDCSNs model. In Section 2, some preliminaries are presented. In Section 3, some new stability principles for the global stability of MSRDCSNs are established. We provide a systematic method to construct the global Lyapunov function of MSRDCSNs by combining graph theory and the Lyapunov second method. The findings show that, if each vertex system has a globally stable equilibrium and possesses a global Lyapunov function $V_{k}$, then the global Lyapunov function for the MSRDCSNs can be systematically produced by individual $V_{k}$. An example is provided in Section 4 . Section 5 is conclusion.

Notations: for convenience, we sometimes write $\mathbf{v}, \mathbf{v}_{\mathbf{k}}$ and $\mathbf{v}_{\mathbf{j}}$ as $\mathbf{v}(t, \mathbf{x}), \mathbf{v}_{\mathbf{k}}(t, \mathbf{x})$ and $\mathbf{v}_{\mathbf{j}}(t, \mathbf{x})$, respectively.

\section{Preliminaries}

Let $\left(\Omega, \mathscr{F}, \mathscr{F}_{t}, \mathbb{P}\right)$ be a complete probability space with a filtration $\left\{\mathscr{F}_{t}\right\}_{t \geq t_{0}}$ satisfying the usual conditions. $W(\cdot)$ be a $m$ dimensional Brownian motion defined on the complete probability space. Let $\{\gamma(t), t \geq 0\}$ be a right-continuous Markov process on the probability space which takes values in the finite space $\mathbb{S}=\{1,2, \ldots, \tilde{N}\}$ with generator $\Gamma=\left(\pi_{k j}\right)(k, j \in \mathbb{S})$ given by

$$
P\{\gamma(t+\Delta)=j \mid \gamma(t)=k\}= \begin{cases}\pi_{k j} \Delta+o(\Delta) & \text { if } k \neq j, \\ 1+\pi_{k k} \Delta+o(\Delta) & \text { if } k=j\end{cases}
$$

where $\Delta>0$ and $\lim _{\Delta \rightarrow 0} o(\Delta) / \Delta=0, \pi_{k j} \geq 0$ is the transition rate from $k$ to $j$ if $k \neq j$ and $\pi_{k k}=-\sum_{j \neq k} \pi_{k j}$. We assume that the Markov chain $\gamma(\cdot)$ is independent of the Brownian motion $W(\cdot)$. A general stochastic reaction-diffusion system with Markovian switching reads

$$
\begin{aligned}
& d \mathbf{v}(t, \mathbf{x})= {[\rho(\gamma(t)) \Delta \mathbf{v}(t, \mathbf{x})+f(t, \mathbf{x}, \mathbf{v}(t, \mathbf{x}), \gamma(t))] d t } \\
&+g(t, \mathbf{x}, \mathbf{v}(t, \mathbf{x}), \gamma(t)) d W(t), \quad(t, \mathbf{x}, \gamma(t)) \in \mathbb{R}_{t_{0}}^{+} \times G \times \mathbb{S} \\
& \mathbf{v}\left(t_{0}, \mathbf{x}\right)= \varphi(\mathbf{x}), \quad \mathbf{x} \in G \\
& \frac{\partial \mathbf{v}(t, \mathbf{x})}{\partial \mathscr{N}}=0, \quad(t, \mathbf{x}) \in \mathbb{R}_{t_{0}}^{+} \times \partial G
\end{aligned}
$$

where $\Delta \mathbf{v}(t, \mathbf{x}) \triangleq\left(\sum_{k=1}^{r} \frac{\partial}{\partial x_{k}}\left[D_{1 k}(t, \mathbf{x}, \mathbf{v}(t, \mathbf{x})) \frac{\partial u_{i}}{\partial x_{k}}\right], \ldots, \sum_{k=1}^{r} \frac{\partial}{\partial x_{k}}\left[D_{n k}(t, \mathbf{x}, \mathbf{v}(t, \mathbf{x})) \frac{\partial u_{i}}{\partial x_{k}}\right]\right)^{T}, G=\left\{\mathbf{x}=\left(x_{1}, x_{2}, \ldots, x_{r}\right)^{T}:\right.$ $\|\mathbf{x}\| l<+\infty\} \subset \mathbb{R}^{r}, \rho=\operatorname{diag}\left(\rho_{1}, \rho_{2}, \ldots, \rho_{n}\right)$ is the diffusion-matrix, $\rho_{n} \geq 0$ is constant; $f: \mathbb{R}_{+} \times G \times \mathbb{R}^{n} \times \mathbb{S} \rightarrow \mathbb{R}^{n}$ and $g: \mathbb{R}_{+} \times G \times \mathbb{R}^{n} \times \mathbb{S} \rightarrow \mathbb{R}^{n \times m}$ are both Borel measurable functions. $D_{i k}(t, \mathbf{x}, \mathbf{v}(t, \mathbf{x})) \geq 0$ is smooth enough. $\mathscr{N}$ is the unit normal vector of $\partial G$. Initial data $\mathbf{v}\left(t_{0}, \mathbf{x}\right)=\mathbf{v}_{0}=\boldsymbol{\varphi}(\mathbf{x})$ is suitably smooth known function and $\gamma\left(t_{0}\right)=\gamma_{0}$, where $\gamma_{0}$ is an $\mathbb{S}$-valued $\mathscr{F}_{t_{0}}$-measurable random variable. $\|\cdot\|$ stands for vector norm.

(Assumption 1) Function $g(t, \mathbf{x}, \mathbf{v}(t, \mathbf{x}), \gamma(t))$ satisfies the integral linear growth condition and $f, g$ meet Lipschitz condition, that is, there exists a constant $L>0$ such that for $\forall i \in \mathbb{S}$

$$
\begin{aligned}
& \|g(t, \mathbf{x}, \mathbf{v}(t, \mathbf{x}), i)\|_{G} \leq L(1+\|\mathbf{v}\|) \\
& \left\|g\left(t, \mathbf{x}, \mathbf{v}_{\mathbf{1}}(t, \mathbf{x}), i\right)-g\left(t, \mathbf{x}, \mathbf{v}_{\mathbf{2}}(t, \mathbf{x}), i\right)\right\|_{G} \leq L\left\|\mathbf{v}_{\mathbf{1}}-\mathbf{v}_{\mathbf{2}}\right\|_{G} \\
& \left\|f\left(t, \mathbf{x}, \mathbf{v}_{\mathbf{1}}(t, \mathbf{x}), i\right)-f\left(t, \mathbf{x}, \mathbf{v}_{\mathbf{2}}(t, \mathbf{x}), i\right)\right\|_{G} \leq L\left\|\mathbf{v}_{\mathbf{1}}-\mathbf{v}_{\mathbf{2}}\right\|_{G}
\end{aligned}
$$

where $\|\mathbf{v}(\cdot, \mathbf{x})\|_{G} \triangleq\left|\int_{G} \mathbf{v}(\cdot, \mathbf{x}) d \mathbf{x}\right|$.

The existence and uniqueness of the solution for system (1) can refer to the relevant conclusions in [22], because stochastic reaction-diffusion systems can be transformed, via semi-group method, into abstract differential systems in Banach space including infinite linear operator and nonlinear term. Suppose that $f(t, \mathbf{x}, 0, i) \equiv 0$ and $g(t, \mathbf{x}, 0, i) \equiv 0, t \geq$ $t_{0}$, which means $\mathbf{v}(t, \mathbf{x})=0$ is a trivial solution of (1).

Let the mathematical expectation with respect to the given probability measure $P$ be denoted by $\mathbb{E}(\cdot)$. Let $|\cdot|$ denote the Euclidean norm for vectors or the trace norm for matrices. We shall use the notations $\mathbb{S}_{\delta}^{n}=\left\{\xi: G \rightarrow \mathbb{R}^{n}:\left|\int_{G} \xi(\mathbf{x}) d \mathbf{x}\right|<\delta\right\}$ and $\mathbb{R}_{+}^{n}=\left\{x \in \mathbb{R}^{n}: x_{k}>0, i=1,2, \ldots, n\right\}$. Some definitions on the stochastic stability of the trivial solution are given as follows.

Definition 1. If for every triple of $\forall \varepsilon_{1} \in(0,1), \forall \varepsilon_{2}>0$ and $t_{0} \geq 0, \ni \delta=\delta\left(\varepsilon_{1}, \varepsilon_{2}, t_{0}\right)>0$ such that

$$
\mathbb{P}\left\{\left\|\mathbf{v}\left(t, \mathbf{x}, t_{0}, \mathbf{v}_{0}, i\right)\right\|_{G}<\varepsilon_{2}, t \geq t_{0}\right\} \geq 1-\varepsilon_{1}
$$

holds for any $\left(\mathbf{v}_{0}, i\right) \in \mathbb{S}_{\delta} \times \mathbb{S}$, the trivial solution of system (1) is said to be stochastically stable or stable in probability. Otherwise, the trivial solution is said to be unstable in probability. 
Definition 2. The trivial solution of Eq. (1) is said to be stochastically asymptotically stable if it is stochastically stable and, moreover, for $\forall \varepsilon \in(0,1)$ and $t_{0} \geq 0$, there exists a $\delta_{0}=\delta_{0}\left(\varepsilon, t_{0}\right)>0$ such that

$$
\mathbb{P}\left\{\lim _{t \rightarrow \infty}\left\|\mathbf{v}\left(t, \mathbf{x}, t_{0}, \mathbf{v}_{0}, i\right)\right\|_{G}=0\right\} \geq 1-\varepsilon
$$

for any $\left(\mathbf{v}_{0}, i\right) \in \mathbb{S}_{\delta_{0}} \times \mathbb{S}$.

Definition 3. The trivial solution of Eq. (1) is said to be stochastically globally asymptotically stable if it is stochastically stable and, moreover, for $\forall \delta>0$ and $\left(\mathbf{v}_{0}, i\right) \in \mathbb{S}_{\delta} \times \mathbb{S}$

$$
\mathbb{P}\left\{\lim _{t \rightarrow \infty}\left\|\mathbf{v}\left(t, \mathbf{x}, t_{0}, \mathbf{v}_{0}, i\right)\right\|_{G}=0\right\}=1 .
$$

Definition 4. The trivial solution of system (1) is said to be almost surely exponentially stable if there is, for any $\left(\mathbf{v}_{0}, i\right) \in$ $\mathbb{S}_{\delta} \times \mathbb{S}$

$$
\lambda \triangleq \lim _{t \rightarrow \infty} \sup \frac{1}{t} \lg \left\|\mathbf{v}\left(t, \mathbf{x}, t_{0}, \mathbf{v}_{0}, i\right)\right\|_{G}<0 \quad \text { a.s. }
$$

where $\lambda$ is called as Lyapunov exponent of the solution for system (1). Therefore, the trivial solution of system (1) is almost surely exponentially stable if and only if $\lambda<0$.

Definition 5. If $\mu(\cdot) \in C[[0, r], \mathbb{R}]$ is a strictly increasing function and $\mu(0)=0$, function $\mu$ is said to be class $\mathcal{K}$ function. Denote $\mu \in \mathcal{K}$ concisely. If $\mu(\cdot) \in C\left[\mathbb{R}^{+}, \mathbb{R}^{+}\right]$and $\mu \in \mathcal{K}, \lim _{r \rightarrow+\infty} \mu(r)=+\infty$, then $\mu \in \mathcal{K} R$.

A continuous function $V(t, \xi, i)$ is said to be positive-definite if $V(t, 0, i)=0$ for $i \in \mathbb{S}$ and for some $\mu \in \mathcal{K}$, $V(t, \xi, i) \geq \mu(|\xi|)$. Write $C^{1,2}\left(\mathbb{R}_{+} \times \mathbb{R}^{n} \times \mathbb{S} ; \mathbb{R}_{+}\right)$for the family of all nonnegative functions $V(t, \xi, i)$ on $\mathbb{R}_{+} \times \mathbb{R}^{n} \times \mathbb{S}$ that are continuously twice differentiable in $\xi$ and once in $t$ for all $i \in \mathbb{S}$. If $V(t, \xi, i) \in C^{1,2}\left(\mathbb{R}_{+} \times \mathbb{R}^{n} \times \mathbb{S} ; \mathbb{R}_{+}\right)$, then define an operator $\mathcal{L V}(t, \xi, i)$ from $\mathbb{R}_{+} \times \mathbb{R}^{n} \times \mathbb{S}$ to $\mathbb{R}$ with respect to (1) by

$$
\begin{aligned}
\mathscr{L} V(t, \xi, i)= & V_{t}(t, \xi, i)+V_{\xi}^{T}(t, \xi, i) f(t, \mathbf{x}, \xi, i)+\frac{1}{2} \operatorname{Trace}\left[g^{T}(t, \mathbf{x}, \xi, i) V_{\xi \xi}(t, \xi, i) g(t, \mathbf{x}, \xi, i)\right] \\
& +\sum_{j=1}^{N} \gamma_{k j} V(t, \xi, j)
\end{aligned}
$$

where

$$
V_{t}(t, \xi, i)=\frac{\partial V(t, \xi, i)}{\partial t}, \quad V_{\xi}^{T}(t, \xi, i)=\left(\frac{\partial V(t, \xi, i)}{\partial \xi_{1}}, \ldots, \frac{\partial V(t, \xi, i)}{\partial \xi_{n}}\right)
$$

and

$$
V_{\xi \xi}(t, \xi, i)=\left(\frac{\partial^{2} V(t, \xi, i)}{\partial \xi_{k} \partial \xi_{j}}\right)_{n \times n}
$$

Applying the generalized Itô formula to $\int_{G} V(t, \mathbf{v}(t, \mathbf{x}), \gamma(t)) d \mathbf{x}$ along system (1) gives for $\forall t \geq t_{0}$

$$
\begin{aligned}
\int_{G} V(t, \mathbf{v}(t, \mathbf{x}), \gamma(t)) d \mathbf{x}= & \int_{G} V\left(t_{0}, \mathbf{v}_{0}, \gamma_{0}\right) d \mathbf{x}+\int_{G} \int_{0}^{t}[\mathcal{L} V(s, \mathbf{v}(s, \mathbf{x}), \gamma(s)) \\
& \left.+V_{\mathbf{v}}^{T}(s, \mathbf{v}, \gamma(s)) \Delta \mathbf{v}(s, \mathbf{x})\right] d s d \mathbf{x}+\int_{G} \int_{0}^{t} V_{\mathbf{v}}^{T}(s, \mathbf{v}) g(s, \mathbf{x}, \mathbf{v}(s, \mathbf{x}), \gamma(s)) d W(s) d \mathbf{x} .
\end{aligned}
$$

The existence of function $V(t, \mathbf{v}(t, \mathbf{x}), i) \in C^{1,2}\left(\mathbb{R}_{+} \times \mathbb{R}^{n} \times \mathbb{S} ; \mathbb{R}_{+}\right)$and another conditions in the classical Lyapunov theorem on the stability of (1) are needed. For convenience, similarly, we give the following definitions:

Definition 6. Function $V \in C^{1,2}\left(\mathbb{R}_{+} \times \mathbb{R}^{n} ; \mathbb{R}_{+}\right)$is called as Lyapunov-A function for (1), if $\mathscr{L} \int_{G} V(t, \mathbf{v}, i) d \mathbf{x} \leq 0$, and is called as Lyapunov-B function for (1), if $\mathcal{L} \int_{G} V(t, \mathbf{v}, i) d \mathbf{x} \leq-b \int_{G} V(t, \mathbf{v}, i) d \mathbf{x}$ in which $b>0$.

The following basic concepts and theorems on graph theory can be found in [7]. A directed graph $q=(\mathcal{V}, E)$ contains a set $\mathcal{V}=\{1,2, \ldots, n\}$ of vertices and a set $E$ of arcs $(i, j)$ leading from initial vertex $i$ to terminal vertex $j$. A subgraph $\mathscr{H}$ of $g$ is said to be spanning if $\mathscr{H}$ and $g$ have the same vertex set. A digraph $g$ is weighted if each $\operatorname{arc}(j, i)$ is assigned a positive weight $a_{k j}$. Here $a_{k j}>0$ if and only if there exists an arc from vertex $j$ to vertex $i$ in $g$. The weight $W(g)$ of $g$ is the product of the weights on all its arcs. A directed path $\mathcal{P}$ in $g$ is a subgraph with distinct vertices $\left\{i_{1}, i_{2}, \ldots, i_{m}\right\}$ such that its set of arcs is $\left\{\left(i_{k}, i_{k+1}\right): k=1,2, \ldots, m-1\right\}$. If $i_{m}=i_{1}$, we call $\mathcal{P}$ a directed cycle. A connected subgraph $\mathcal{T}$ is a tree if it contains no 
cycles. A tree $\mathcal{T}$ is rooted at vertex $i$, called the root, if $i$ is not a terminal vertex of any arcs, and each of the remaining vertices is a terminal vertex of exactly one arc. A digraph $g$ is strongly connected if, for any pair of distinct vertices, there exists a directed path from one to the other. Given a weighted digraph $g$ with $n$ vertices, define the weight matrix $A=\left(a_{i j}\right)_{n \times n}$ whose entry $a_{k j}$ equals the weight of arc $(j, i)$ if it exists, and 0 otherwise. Denote the directed graph with weight matrix $A$ as $(\mathcal{G}, A)$. A weighted digraph $(\mathcal{G}, A)$ is said to be balanced if $W(\mathcal{C})=W(-\mathcal{C})$ for all directed cycles $\mathcal{C}$. Here, $-\mathcal{C}$ denotes the reverse of $\mathcal{C}$ and is constructed by reversing the direction of all arcs in $\mathcal{C}$. For a unicyclic graph $\mathcal{Q}$ with cycle $\mathcal{C}_{\mathcal{Q}}$, let $\tilde{\mathcal{Q}}$ be the unicyclic graph obtained by replacing $\mathcal{C}_{\mathcal{Q}}$ with $-\mathcal{C}_{\mathcal{Q}}$. Suppose that $(\mathcal{G}, A)$ is balanced, then $W(\mathcal{Q})=W(\tilde{Q})$. The Laplacian matrix of $(G, A)$ is defined as

$$
L=\left(\begin{array}{cccc}
\sum_{k \neq 1} a_{1 k} & -a_{12} & \cdots & -a_{1 n} \\
-a_{21} & \sum_{k \neq 2} a_{2 k} & \cdots & -a_{2 n} \\
\vdots & \vdots & \ddots & \vdots \\
-a_{n 1} & -a_{n 2} & \cdots & \sum_{k \neq n} a_{n k}
\end{array}\right) .
$$

Let $c_{k}$ denote the cofactor of the $i$ th diagonal element of $L$.

Lemma 1 (Kirchhoff's Matrix Tree Theorem). Assume $n \geq 2$. Then

$$
c_{k}=\sum_{\mathcal{T} \in \mathbb{T}_{k}} W(\mathcal{T}), \quad k=1,2, \ldots, N
$$

where $\mathbb{T}_{k}$ is the set of all spanning trees $\mathcal{T}$ of $(g, A)$ that are rooted at vertex $i$. In particular, if $(g, A)$ is strongly connected, then $c_{k}>0$ for $c_{k}=1,2, \ldots, n$.

Lemma 2 ([8]). Assume $n \geq 2$. Let $c_{k}$ be given in (5). Then the following identity holds:

$$
\sum_{k, j=1}^{n} c_{k} a_{k j} F_{k j}\left(x_{k}, x_{j}\right)=\sum_{\mathcal{Q} \in \mathbb{Q}} W(\mathcal{Q}) \sum_{(k, j) \in E\left(C_{Q}\right)} F_{k j}\left(x_{k}, x_{j}\right) .
$$

Here $F_{k j}\left(x_{k}, x_{j}\right), 1 \leq k, j \leq n$, are arbitrary functions, $\mathbb{Q}$ is the set of all spanning unicyclic graphs of $(q, A), W(\mathcal{Q})$ is the weight of $\mathcal{Q}$, and $C_{\mathbb{Q}}$ denotes the directed cycle of $\mathcal{Q}$.

Lemma 3. Under Assumption 1,

$$
\mathbb{P}\left\{\left\|\mathbf{v}\left(t, \mathbf{x}, t_{0}, \mathbf{v}_{0}, i\right)\right\|_{G} \neq 0, t \geq t_{0}\right\}=1
$$

for all $\left(t_{0}, \mathbf{v}_{0}, \gamma_{0}\right) \in \mathbb{R}_{+} \times\left(\mathbb{R}^{n}-0\right) \times \mathbb{S}$. That is, almost any trajectory of (1) starting from a non-zero state will never be zero.

Proof. If the lemma was false, there would exist some $t_{0} \geq 0, \mathbf{v}_{0} \neq 0$ and $i \in \mathbb{S}$ such that $\mathbb{P}\{\tau<\infty\}>0$, where $\tau$ is the first time to reach zero for the corresponding solution, that is,

$$
\tau=\inf \left\{t \geq t_{0}, \int_{G} \mathbf{v}\left(t, \mathbf{x}, t_{0}, \mathbf{v}_{0}, \gamma_{0}\right) d \mathbf{x}=0\right\} .
$$

It is not difficult to find an integer $k>t_{0} \vee\left(1+\left\|\mathbf{v}_{0}\right\|\right)$ sufficiently large for $\mathbb{P}\{B\}>0$, where $B=\{\tau<T, T$ is the time upper bound and $\|\mathbf{v}(t, \mathbf{x})\| \leq k-1$, for $\left.\forall(t, \mathbf{x}) \in\left[t_{0}, \tau\right] \times G\right\}$. While in view of the linear growth conditions there is positive constant $L_{k}$ such that, for any $\|\mathbf{v}(t, \mathbf{x})\| \geq k, \forall(t, \mathbf{x}, i) \in\left[t_{0}, \tau\right] \times G \times \mathbb{S}$,

$$
\|f(t, \mathbf{x}, \mathbf{v}(t, \mathbf{x}), i)\|_{G} \vee\|g(t, \mathbf{x}, \mathbf{v}(t, \mathbf{x}), i)\|_{G} \leq L_{k}(1+\|\mathbf{v}\|) .
$$

Let $V(t, \mathbf{v}, i)=\|\mathbf{v}\|^{-1}$, we calculate, for $\|\mathbf{v}(t, \mathbf{x})\| \geq k, \forall(t, \mathbf{x}, i) \in\left[t_{0}, \tau\right] \times G \times \mathbb{S}$,

$$
\begin{aligned}
\mathscr{L} \int_{G} V(t, \mathbf{v}(t, \mathbf{x}), i) d \mathbf{x}= & \int_{G} \mathcal{L} V(t, \mathbf{v}(t, \mathbf{x}), i) d \mathbf{x} \\
= & \int_{G}\left[-\|\mathbf{v}\|^{-3} \mathbf{v}^{T} f(t, \mathbf{x}, \mathbf{v}, i)-\|\mathbf{v}\|^{-3} \mathbf{v}^{T} \rho(i) \Delta \mathbf{v}(t, \mathbf{x})\right. \\
& \left.+\frac{1}{2}\left(-\|\mathbf{v}\|^{-3}\|g(t, \mathbf{x}, \mathbf{v}, i)\|^{2}+3\|\mathbf{v}\|^{-5}\left\|\mathbf{v} T^{T} g(t, \mathbf{x}, \mathbf{v}, i)\right\|^{2}\right)\right] d \mathbf{x} \\
\leq & \int_{G}\left[\|\mathbf{v}\|^{-1}\|f(t, \mathbf{x}, \mathbf{v}, i)\|+\|\mathbf{v}\|^{-1}\|g(t, \mathbf{x}, \mathbf{v}, i)\|^{2}\right] d \mathbf{x} \\
\leq & \int_{G}\left[L_{k}\|\mathbf{v}\|^{-1}+L_{k}^{2}\|\mathbf{v}\|^{-1}\right] d \mathbf{x}=|G| L_{k}\left(1+L_{k}\right)\|\mathbf{v}\|^{-1} .
\end{aligned}
$$


For any $\varepsilon \in\left(0,\left\|\mathbf{v}_{0}\right\|\right)$, define a stopping time $\tau_{\varepsilon}=\inf \left\{t \geq t_{0}:\|\mathbf{v}\| \notin(\varepsilon, k)\right\}$. Applying the Itô formula gives

$$
\begin{aligned}
& \mathbb{E}\left[e^{-L_{k}\left(1+L_{k}\right)\left(\tau_{\varepsilon} \wedge k\right)} \int_{G} V\left(\tau_{\varepsilon} \wedge k, \mathbf{v}\left(\tau_{\varepsilon} \wedge k, \mathbf{x}\right), i\right) d \mathbf{x}\right]=\left\|\mathbf{v}_{0}\right\|^{-1} e^{-L_{k}\left(1+L_{k}\right) t_{0}}|G| \\
& \quad+\mathbb{E} \int_{t_{0}}^{\tau_{\varepsilon} \wedge k} e^{-L_{k}\left(1+L_{k}\right) s}\left[-|G| L_{k}\left(1+L_{k}\right)\|\mathbf{v}(s, \mathbf{x})\|^{-1}+\int_{G} V(s, \mathbf{v}(s, \mathbf{x}), \gamma(s)) d \mathbf{x}\right] d s \leq\left\|\mathbf{v}_{0}\right\|^{-1} e^{-L_{k}\left(1+L_{k}\right) t_{0}}|G| .
\end{aligned}
$$

Note that for $\omega \in B$ we have $\tau_{\varepsilon} \leq k$ and $\left\|\mathbf{v}\left(\tau_{\varepsilon} \leq k, \mathbf{x}\right)\right\|=\varepsilon$, then we obtain

$$
\mathbb{E}\left[e^{-L_{k}\left(1+L_{k}\right) k} \varepsilon^{-1}|G| X_{B}\right] \leq\left\|\mathbf{v}_{0}\right\|^{-1} e^{-L_{k}\left(1+L_{k}\right) t_{0}}|G| .
$$

Thus,

$$
\mathbb{P}\{B\} \leq \varepsilon\left\|\mathbf{v}_{0}\right\|^{-1} e^{-L_{k}\left(1+L_{k}\right)\left(k-t_{0}\right)}|G| .
$$

Letting $\varepsilon \rightarrow 0$ yields $\mathbb{P}\{B\}=0$, which is in contradiction with the definition of $\mathbb{P}\{B\}>0$. The proof is complete.

\section{Global stability analysis for Markovian switching coupled reaction-diffusion systems on networks}

To begin with our main results, we will give a MSRDCSNs represented by digraph $q$ with $N$ vertices, $N \geq 2$. In $i$ th vertex it is assigned a stochastic reaction-diffusion system with Markovian switching

$$
\begin{aligned}
d \mathbf{v}_{k}(t, \mathbf{x})= & {\left[\rho_{k}(\gamma(t)) \Delta \mathbf{v}_{k}(t, \mathbf{x})+f_{k}\left(t, \mathbf{x}, \mathbf{v}_{k}(t, \mathbf{x}), \gamma(t)\right)\right] d t } \\
& +g_{k}\left(t, \mathbf{x}, \mathbf{v}_{k}(t, \mathbf{x}), \gamma(t)\right) d W_{k}(t), \quad(t, \mathbf{x}, \gamma(t)) \in \mathbb{R}_{t_{0}}^{+} \times \Omega \times \mathbb{S}
\end{aligned}
$$

where $\mathbf{v}_{k}(t, \mathbf{x}) \in \mathbb{R}^{n_{k}}, f_{k}: \mathbb{R}_{+} \times G \times \mathbb{R}^{n_{k}} \times \mathbb{S} \rightarrow \mathbb{R}^{n_{k}}$ and $g_{k}: \mathbb{R}_{+} \times G \times \mathbb{R}^{n_{k} \times m_{i}} \times \mathbb{S} \rightarrow \mathbb{R}^{n_{k} \times m}$. If these systems are coupled, let

$$
H_{k j}: \mathbb{R}^{n_{k}} \times \mathbb{R}^{n_{j}} \times \mathbb{R} \times \mathbb{S} \rightarrow \mathbb{R}^{m_{i}}, \quad N_{k j}: \mathbb{R}^{n_{k}} \times \mathbb{R}^{n_{j}} \times \mathbb{R} \times \mathbb{S} \rightarrow \mathbb{R}^{n_{k} \times m}, \quad k, j=1,2, \ldots, N
$$

represent the influence of vertex $j$ on vertex $i$, and $H_{k j}=N_{k j}=0$ if there exists no $\operatorname{arc}$ from $j$ to $i$ in $g$. Then, by replacing $f_{k}$ and $g_{k}$ with $f_{k}+\sum_{j=1}^{n} H_{k j}$ and $g_{k}+\sum_{j=1}^{n} N_{k j}$, we get the following stochastic coupled system on graph $g$ :

$$
\begin{aligned}
d \mathbf{v}_{k}(t, \mathbf{x})= & {\left[\rho_{k}(i) \Delta \mathbf{v}_{k}(t, \mathbf{x})+f_{k}\left(t, \mathbf{x}, \mathbf{v}_{k}(t, \mathbf{x}), i\right)+\sum_{j=1}^{N} H_{k j}\left(\mathbf{v}_{k}, \mathbf{v}_{j}, t, i\right)\right] d t } \\
& +\left[g_{k}\left(t, \mathbf{x}, \mathbf{v}_{k}(t, \mathbf{x}), i\right)+\sum_{j=1}^{N} N_{k j}\left(\mathbf{v}_{k}, \mathbf{v}_{j}, t, i\right)\right] d W(t), \quad(t, \mathbf{x}, i) \in \mathbb{R}_{t_{0}}^{+} \times G \times \mathbb{S} \\
\mathbf{v}_{k}\left(t_{0}, \mathbf{x}\right)= & \boldsymbol{\varphi}_{\mathbf{k}(\mathbf{x})}, \quad \mathbf{x} \in G, \gamma\left(t_{0}\right)=\gamma_{0} \\
\frac{\partial \mathbf{v}_{k}(t, \mathbf{x})}{\partial \mathscr{N}}= & 0, \quad(t, \mathbf{x}) \in \mathbb{R}_{t_{0}}^{+} \times \partial G .
\end{aligned}
$$

Without loss of generality, we suppose that functions $f_{k}, g_{k}, H_{k j}$ and $N_{k j}$ are such that initial-value problems to (7) and (8) have unique solution and trivial solution $\mathbf{v}(t, \mathbf{x})=\left(\mathbf{v}_{1}, \ldots, \mathbf{v}_{n}\right)=0$. Functions $f_{k}$ and $g_{k}$ meet Lipschitz conditions and linear growth conditions with constant $L>0$. For $V_{k}\left(t, \mathbf{v}_{k}, i\right) \in C^{1,2}\left(\mathbb{R}_{+} \times \mathbb{R}^{n_{k}} \times \mathbb{S} ; \mathbb{R}_{+}\right)$, define a differential operator $\mathcal{L} V_{k}\left(t, \mathbf{v}_{k}, i\right)$ associated with the $i$ th equation of $(8)$ by

$$
\begin{aligned}
\mathcal{L} V_{k}\left(t, \mathbf{v}_{k}, i\right) \triangleq & \frac{\partial V_{k}\left(t, \mathbf{v}_{k}, i\right)}{\partial t}+\left(\frac{\partial V_{k}\left(t, \mathbf{v}_{k}, i\right)}{\partial \mathbf{v}_{k}}\right)^{T}\left[f_{k}\left(t, \mathbf{x}, \mathbf{v}_{k}(t, \mathbf{x}), i\right)+\sum_{j=1}^{N} H_{k j}\left(\mathbf{v}_{k}, \mathbf{v}_{j}, t, i\right)\right] \\
& +\frac{1}{2} \operatorname{Trace}\left\{\left[g_{k}\left(t, \mathbf{x}, \mathbf{v}_{k}(t, \mathbf{x}), i\right)+\sum_{j=1}^{N} N_{k j}\left(\mathbf{v}_{k}, \mathbf{v}_{j}, t, i\right)\right]^{T}\left(V_{k}\left(t, \mathbf{v}_{k}\right)\right)_{\mathbf{v}_{k} \mathbf{v}_{j}}^{\prime \prime}\right. \\
& \left.\times\left[g_{k}\left(t, \mathbf{x}, \mathbf{v}_{k}(t, \mathbf{x}), i\right)+\sum_{j=1}^{N} N_{k j}\left(\mathbf{v}_{k}, \mathbf{v}_{j}, t, i\right)\right]\right\}+\sum_{j=1}^{N} \gamma_{k j} V(t, \mathbf{v}, j) .
\end{aligned}
$$

Theorem 1. Suppose that the following conditions hold.

A1. Assume that there exist positive functions $V_{k}(t, \xi, i) \in C^{1,2}\left(\mathbb{R}_{+} \times \mathbb{R}^{n_{k}} \times \mathbb{S} ; \mathbb{R}_{+}\right)$, functions $F_{k j}\left(\mathbf{v}_{k}, \mathbf{v}_{j}, t\right)$ and constants $a_{k j} \geq 0$ satisfying 
(I) there exist $\mu_{1}, \mu_{2} \in \mathcal{K} R$ such that

$$
\begin{aligned}
& \mu_{1}\left(\left\|\mathbf{v}_{k}\right\|\right) \leq \int_{G} V_{k}\left(t, \mathbf{v}_{k}, i\right) d \mathbf{x} \leq \mu_{2}\left(\left\|\mathbf{v}_{k}\right\|\right), \\
& \mathcal{L} \int_{G} V_{k}\left(t, \mathbf{v}_{k}, i\right) d \mathbf{x} \leq \sum_{j=1}^{n} a_{k j} F_{k j}\left(\mathbf{v}_{k}, \mathbf{v}_{j}, t\right), \\
& t \geq t_{0}, \quad k=1,2, \ldots, N,
\end{aligned}
$$

hold for $\forall\left(t, \mathbf{v}_{k}(t, x), i\right) \in\left[t_{0}, \infty\right) \times \mathbb{s}_{h}^{n_{k}} \times \mathbb{S}$, where $\mathbf{v}_{k}(t, \cdot) \in \mathbb{s}_{h}^{n_{k}}=\left\{\zeta: G \rightarrow \mathbb{R}^{n_{k}} \| \int_{G} \zeta(\mathbf{x}) d \mathbf{x} \mid<h\right\}$;

(II) $V_{k}(t, \xi, i)$ is separated as to variables $\xi(k \in N)$ for $i \in \mathbb{S}$;

(III) $\frac{\partial^{2} V_{k}(t, \xi, i)}{\partial \xi^{2}} \geq 0, k \in N,(t, \xi) \in \mathbb{R}_{+} \times \mathbb{R}^{n}$ for $i \in \mathbb{S}$;

A2. Along each directed cycle $\mathcal{C}$ of the weighted digraph $(g, A)$ in which $A=\left(a_{k j}\right)_{n \times n}$, there is

$$
\sum_{(i, j) \in E(\mathcal{C})} F_{k j}\left(\mathbf{v}_{k}, \mathbf{v}_{j}, t\right) \leq 0, \quad t \geq t_{0} .
$$

Then function $V(t, \mathbf{v}, i) \triangleq \sum_{i=1}^{n} c_{k} V_{k}\left(t, \mathbf{v}_{k}, i\right)$ is a Lyapunov-A function for (8), in which $c_{k}$ is defined as (5). Furthermore, the trivial solution of $(8)$ is stochastically stable. In addition, if more condition is added.

A3. If $V_{k}(t, \xi, i)$ is radial unbounded, then the trivial solution of (8) is globally asymptotically stable in probability.

Proof. We first prove that the trivial solution of system (8) is stochastically stable.

For $\forall \varepsilon_{1} \in(0,1)$ and $\varepsilon_{2} \geq 0$, suppose $\varepsilon_{2}<h$. Since $V_{k}(t, \xi, i)$ is continuous and $V_{k}\left(t_{0}, 0, i\right)=0$, there is $\delta=\delta$ $\left(\varepsilon_{1}, \varepsilon_{1}, t_{0}\right)>0$, such that

$$
\frac{1}{\varepsilon_{1}} \sup _{\mathbf{v}(t, \mathbf{x}) \in \mathbb{S}_{\delta}^{n}} \int_{G} V\left(t_{0}, \mathbf{v}(t, \mathbf{x}), i\right) d \mathbf{x} \leq \mu^{*}\left(\varepsilon_{2}\right)
$$

where $n=n_{1}+n_{2}+\cdots+n_{N}, \mu^{*} \in \mathcal{K} R$.

It follows from Condition (I) and (12) that $\delta<\varepsilon_{2}$. Denote $\overline{\mathbf{v}}(t)=\int_{G} \mathbf{v}\left(t, \mathbf{x}, t_{0}, \mathbf{v}_{0}, i\right) d \mathbf{x}$ for $\forall\left(\mathbf{v}_{0}, i\right) \in \mathbb{S}_{\delta}^{n} \times \mathbb{S}$. Let $\tau$ be the first escape time of $\overline{\mathbf{v}}(t)$ from $\mathbb{S}_{\varepsilon_{2}}^{n}$, that is

$$
\tau=\inf \left\{t \geq t_{0} \mid \overline{\mathbf{v}}(t) \notin \mathbb{S}_{\varepsilon_{2}}^{n}\right\}
$$

Applying the Itô formula to $\int_{G} V_{k}\left(t, \mathbf{v}_{k}(t, \mathbf{x}), i\right) d \mathbf{x}$ along system (8) gives for $\forall t \geq t_{0}$

$$
\begin{aligned}
\int_{G} V_{k}\left(\tau \wedge t, \mathbf{v}_{k}(\tau \wedge t, \mathbf{x}), \gamma(\tau \wedge t)\right) d \mathbf{x}= & \int_{G} V_{k}\left(t_{0}, \mathbf{v}_{k 0}, \gamma_{0}\right) d \mathbf{x} \\
& +\int_{t_{0}}^{\tau \wedge t} \int_{G} \mathcal{L} V_{k}\left(s, \mathbf{v}_{k}\left(s, \mathbf{v}, t_{0}, \mathbf{v}_{k 0}, i\right)\right) d \mathbf{x} d s \\
& +\int_{t_{0}}^{\tau \wedge t} \int_{G}\left(\frac{\partial V_{k}\left(t, \mathbf{v}_{k}, i\right)}{\partial \mathbf{v}_{k}}\right)^{T} \triangle \mathbf{v}_{k}(s, \mathbf{x}) d \mathbf{x} d s \\
& +\int_{t_{0}}^{\tau \wedge t} \int_{G}\left(\frac{\partial V_{k}\left(t, \mathbf{v}_{k}, i\right)}{\partial \mathbf{v}_{k}}\right)^{T}\left[g_{k}\left(t, \mathbf{x}, \mathbf{v}_{k}\left(t, \mathbf{x}, t_{0}, \mathbf{v}_{k 0}, i\right)\right)\right. \\
& \left.+\sum_{j=1}^{N} N_{k j}\left(\mathbf{v}_{k}, \mathbf{v}_{j}, t, i\right)\right] d \mathbf{x} d W(t) .
\end{aligned}
$$

By Condition A1(II), we have $\frac{\partial^{2} V_{k}(t, \xi)}{\partial \xi_{k} \partial \xi_{j}}=0,(k \neq j, k, j \in N)$. Employing integration by parts and combining Condition A1(II), A1(III) and boundary condition of (8), we obtain

$$
\int_{G}\left(\frac{\partial V_{k}\left(t, \mathbf{v}_{k}, i\right)}{\partial \mathbf{v}_{k}}\right)^{T} \Delta \mathbf{v}_{k}(t, \mathbf{x}) d \mathbf{x} \leq 0 .
$$

Furthermore, due to the continuity of $\frac{\partial V_{k}\left(t, \mathbf{v}_{k}, i\right)}{\partial \mathbf{v}_{k}}$ on $\left[t_{0}, \tau \wedge t\right] \times \mathbb{S}_{h}^{\mathbb{R}_{k}} \times \mathbb{S}$, there must exist constant $L_{1}>0$ such that $\left\|\left(\frac{\partial V_{k}\left(t, \mathbf{v}_{k}, i\right)}{\partial \mathbf{v}_{k}}\right)^{T}\right\| \leq L_{1}$ holds for $(t, \mathbf{v}, i) \in\left[t_{0}, \tau \wedge t\right] \times \mathbb{S}_{h}^{\mathbb{R}^{k_{k}}} \times \mathbb{S}$, in addition, since $g_{k}\left(t, \mathbf{x}, \mathbf{v}_{k}(t, \mathbf{x}), i\right)$ meets integral linear growth condition, we obtain, for $(t, \mathbf{v}(t, \mathbf{x})) \in\left[t_{0}, \tau \wedge t\right] \times \mathbb{S}_{h}^{\mathbb{R}^{n_{k}}}$

$$
\left\|\left(\frac{\partial V_{k}\left(t, \mathbf{v}_{k}, i\right)}{\partial \mathbf{v}_{k}}\right)^{T} g_{k}\left(t, \mathbf{x}, \mathbf{v}_{k}(t, \mathbf{x}), i\right)\right\|_{G} \leq L_{1} L\left(1+\left\|\mathbf{v}_{k}(t, \mathbf{x})\right\|_{G}\right) \leq L_{1} L(1+h)
$$


From Theorem 1.45 of Ref. ([22], pp. 49), we have

$$
\mathbb{E}\left[\int_{t_{0}}^{\tau \wedge t} \int_{G}\left(\frac{\partial V_{k}\left(t, \mathbf{v}_{k}, i\right)}{\partial \mathbf{v}_{k}}\right)^{T}\left(g_{k}\left(t, \mathbf{x}, \mathbf{v}_{k}\left(t, \mathbf{x}, t_{0}, \mathbf{v}_{k 0}\right), i\right)+\sum_{j=1}^{N} N_{k j}\left(\mathbf{v}_{k}, \mathbf{v}_{j}, t, i\right)\right) d \mathbf{x} d W(t)\right]=0 .
$$

On the other hand, by (10), it is derived that

$$
\mathcal{L} \int_{G} V(t, \mathbf{v}, i) d \mathbf{x}=\sum_{i=1}^{n} c_{k} \mathcal{L} \int_{G} V_{k}\left(t, \mathbf{v}_{k}, i\right) d \mathbf{x} \leq \sum_{k, j=1} c_{k} a_{k j} F_{k j}\left(\mathbf{v}_{k}, \mathbf{v}_{j}, t\right) .
$$

Making use of Lemma 2 with weighted digraph $(g, A)$, it yields

$$
\sum_{k, j=1} c_{k} a_{k j} F_{k j}\left(\mathbf{v}_{k}, \mathbf{v}_{j}, t\right)=\sum_{Q \in \mathbb{Q}} W(\mathcal{Q}) \sum_{(i, j) \in E\left(\mathcal{C}_{Q}\right)} F_{k j}\left(\mathbf{v}_{k}, \mathbf{v}_{j}, t\right) .
$$

In view of Condition $A 2$ and the fact $W(\mathcal{Q})>0$, we get

$$
\mathcal{L} \int_{G} V(t, \mathbf{v}, i) d \mathbf{x} \leq \sum_{\mathbb{Q} \in \mathbb{Q}} W(\mathcal{Q}) \sum_{(i, j) \in E\left(C_{Q}\right)} F_{k j}\left(\mathbf{v}_{k}, \mathbf{v}_{j}, t\right) \leq 0 .
$$

Thus $V(t, \mathbf{v}, i)$ is a Lyapunov-A function for (8). Taking the mathematical expectation at the two sides of Eq. (13) and using (15), (16) and (19) we have

$$
\mathbb{E}\left[\int_{G} V(\tau \wedge t, \mathbf{v}(\tau \wedge t, \mathbf{x}), \gamma(\tau \wedge t)) d \mathbf{x}\right] \leq \int_{G} V\left(t_{0}, \mathbf{v}_{0}, \gamma_{0}\right) d \mathbf{x} .
$$

Recalling the fact that $\|\mathbf{v}(\tau \wedge t, \mathbf{x})\|_{G}=\|\mathbf{v}(\tau, \mathbf{x})\|_{G}=\varepsilon_{2}$ if $\tau \leq t$, it is derived

$$
\mathbb{E}\left[\int_{G} V(\tau \wedge t, \mathbf{v}(\tau \wedge t, \mathbf{x}), \gamma(\tau \wedge t)) d \mathbf{x}\right] \geq \mathbb{E}\left[I_{\{\tau<t\}} \int_{G} V(\tau, \mathbf{v}(\tau, \mathbf{x}), \gamma(\tau)) d \mathbf{x}\right] \geq \mu^{*}\left(\varepsilon_{2}\right) \mathbb{P}(\tau \leq t) .
$$

Combining (12) and (19) we get $\mathbb{P}(\tau \leq t) \leq \varepsilon_{1}$. Letting $t \rightarrow \infty$, we infer that

$$
\mathbb{P}(\tau \leq \infty) \leq \varepsilon_{1} .
$$

That is,

$$
\mathbb{P}\left(|\overline{\mathbf{v}}(t)|<\varepsilon_{2}, \forall t \geq t_{0}\right) \geq 1-\varepsilon_{1} .
$$

Namely,

$$
\mathbb{P}\left(\left\|\mathbf{v}\left(t, \mathbf{x}, t_{0}, \mathbf{v}_{0}, i\right)\right\|_{G}<\varepsilon_{2}, t \geq t_{0}\right) \geq 1-\varepsilon_{1}
$$

which implies that the trivial solution of system (8) is stable in probability.

We then prove that the trivial solution of system (8) is globally asymptotically stable in probability. In the following, we only need to prove for $\forall \mathbf{v}_{0}$,

$$
\mathbb{P}\left(\lim _{t \rightarrow \infty}\left\|\mathbf{v}\left(t, \mathbf{x}, t_{0}, \mathbf{v}_{0}, i\right)\right\|_{G}=0\right)=1 .
$$

For $\forall \varepsilon \in(0,1)$, since $V_{k}(t, \xi, i)$ is radial unbounded, we can find $h>\left\|\mathbf{v}_{0}\right\|_{G}$ for $i \in \mathbb{S}$ satisfying

$$
\inf _{t \geq t_{0},\|\mathbf{v}\|_{G} \geq h} \int_{G} V\left(t_{0}, \mathbf{v}(t, \mathbf{x}), i\right) d \mathbf{x} \geq \frac{4}{\varepsilon} \int_{G} V\left(t_{0}, \mathbf{v}_{0}, i\right) d \mathbf{x} .
$$

Define stop-time $\tau_{h}=\inf \left\{t \geq t_{0},\|\overline{\mathbf{v}}(t)\| \geq h\right\}$. Applying Itô formula, similar to get (19), we obtain for $t \geq t_{0}$

$$
\mathbb{E}\left[\int_{G} V\left(\tau_{h} \wedge t, \mathbf{v}\left(\tau_{h} \wedge t, \mathbf{x}\right), \gamma(\tau \wedge t)\right) d \mathbf{x}\right] \leq \int_{G} V\left(t_{0}, \mathbf{v}_{0}, \gamma_{0}\right) d \mathbf{x} .
$$

From (21), we get

$$
\mathbb{E}\left[\int_{G} V\left(\tau_{h} \wedge t, \mathbf{v}\left(\tau_{h} \wedge t, \mathbf{x}\right), \gamma(\tau \wedge t)\right) d \mathbf{x}\right] \geq \frac{4}{\varepsilon} \int_{G} V\left(t_{0}, \mathbf{v}_{0}, \gamma_{0}\right) d \mathbf{x} \mathbb{P}\left\{\tau_{h} \leq t\right\} .
$$

Then, it follows that

$$
\mathbb{P}\left\{\tau_{h} \leq t\right\} \leq \frac{\varepsilon}{4} .
$$


Letting $t \rightarrow \infty$ results in

$$
\mathbb{P}\left\{\tau_{h} \leq \infty\right\} \leq \frac{\varepsilon}{4} .
$$

Therefore

$$
\mathbb{P}\left(|\overline{\mathbf{v}}(t)|<h, \forall t \geq t_{0}\right) \geq 1-\frac{\varepsilon}{4} .
$$

From Theorem 4.2.3 of Ref. ([39], pp. 112-114),

$$
\mathbb{P}(|\overline{\mathbf{v}}(t)|=0) \geq 1-\varepsilon .
$$

Thus, (20) holds owing to the arbitrariness of $\varepsilon$. The proof is complete.

Note that if $(g, A)$ is balanced, then

$$
\sum_{k, j=1} c_{k} a_{k j} F_{k j}\left(\mathbf{v}_{k}, \mathbf{v}_{j}, t\right)=\frac{1}{2} \sum_{\mathcal{Q} \in \mathbb{Q}} W(\mathcal{Q}) \sum_{(k, j) \in E\left(C_{\mathcal{Q}}\right)}\left[F_{j k}\left(\mathbf{v}_{j}, \mathbf{v}_{k}, t\right)+F_{k j}\left(\mathbf{v}_{k}, \mathbf{v}_{j}, t\right)\right] .
$$

In this case, Condition $\mathrm{A} 2$ is replaced by the following:

$$
\sum_{(k, j) \in E\left(C_{Q}\right)}\left[F_{j k}\left(\mathbf{v}_{j}, \mathbf{v}_{k}, t\right)+F_{k j}\left(\mathbf{v}_{k}, \mathbf{v}_{j}, t\right)\right] \leq 0 .
$$

Consequently, we get the following corollary:

Corollary 1. Suppose that $(g, A)$ is balanced. Then the conclusion of Theorem 1 holds if (11) is replaced by (24).

Remark 1. The MSRDCSNs is so complicated that it is very difficult to give the analytical solution. It is important to work on the qualitative analysis of the system. Therefore, how to construct appropriate Lyapunov function is of great importance. The proof shows that, if each vertex system of (8) has a globally stable trivial solution and possesses a Lyapunov function $V_{k}$, then the Lyapunov function for (8) can be systematically constructed by using individual $V_{k}$. In special, when $\rho_{k}=0, m=1$ some examples are given in [12]. Moreover, when $g_{k}=0$ and $N_{k j}=0(k, j=1,2, \ldots, n)$, some examples are given in [8].

Theorem 2. Suppose that the following conditions hold.

B1. Assume that there exist positive functions $V_{k}(t, \xi, i) \in C^{1,2}\left(\mathbb{R}_{+} \times \mathbb{R}^{n_{k}} \times \mathbb{S} ; \mathbb{R}_{+}\right)$, satisfying

(I) there exist $\mu_{1}, \mu_{2} \in \mathcal{K} R$ such that

$$
\mu_{1}\left(\left\|\mathbf{v}_{k}\right\|\right) \leq \int_{G} V_{k}\left(t, \mathbf{v}_{k}, i\right) d \mathbf{x} \leq \mu_{2}\left(\left\|\mathbf{v}_{k}\right\|\right)
$$

hold for $\forall\left(t, \mathbf{v}_{k}(t, \mathbf{x})\right) \in\left[t_{0}, \infty\right) \times \mathbb{s}_{h}^{n_{k}}$, where $\mathbf{v}_{k}(t, \cdot) \in \mathbb{s}_{h}^{n_{k}}=\left\{\zeta: G \rightarrow \mathbb{R}^{n_{k}} \| \int_{G} \zeta(\mathbf{x}) d \mathbf{x} \mid<h\right\}$;

(II) $V_{k}(t, \xi, i)$ is separated as to variables $\xi(k \in N)$ for $i \in \mathbb{S}$;

(III) $\frac{\partial^{2} V_{k}(t, \xi, i)}{\partial \xi^{2}} \geq 0, k \in N,(t, \xi) \in \mathbb{R}_{+} \times \mathbb{R}^{n}$ for $i \in \mathbb{S}$.

B2. There exist functions $F_{k j}\left(\mathbf{v}_{k}, \mathbf{v}_{j}, t\right)$ and constants $a_{k j} \geq 0, b_{k}>0$ such that

$$
\mathcal{L} \int_{G} V_{k}\left(t, \mathbf{v}_{k}, i\right) d \mathbf{x} \leq-b_{k} \int_{G} V_{k}\left(t, \mathbf{v}_{k}, i\right) d \mathbf{x}+\sum_{j=1}^{n} a_{k j} F_{k j}\left(\mathbf{v}_{k}, \mathbf{v}_{j}, t\right),
$$

$t \geq t_{0}$, for $k=1,2, \ldots, N$.

B3. Condition A2 holds, or if $(g, A)$ is balanced and (24) holds.

Then function $V(t, \mathbf{v}, i) \triangleq \sum_{i=1}^{n} c_{k} V_{k}\left(t, \mathbf{v}_{k}, i\right)$ is a Lyapunov-B function for (8), in which $c_{k}$ is defined as (5). Consequently, the trivial solution of (8) is asymptotically stable in probability.

In addition, if more condition is added.

B4. Each $V_{k}(x, t, i)$ satisfies

$$
\lim _{\left\|\mathbf{v}_{k}\right\| \rightarrow \infty} \inf _{t \geq t_{0}} \int_{G} V_{k}\left(t, \mathbf{v}_{k}, i\right) d \mathbf{x}=\infty .
$$

Then the trivial solution of (8) is globally asymptotically stable in probability.

Proof. We can show in the same way as in the proof of Theorem 1 that

$$
\mathcal{L} \int_{G} V(t, \mathbf{v}, i) d \mathbf{x}=\sum_{i=1}^{n} c_{k} \mathcal{L} \int_{G} V_{k}\left(t, \mathbf{v}_{k}, i\right) d \mathbf{x} \leq-b \int_{G} V(t, \mathbf{v}, i) d \mathbf{x},
$$


where $b=\min \left\{b_{1}, b_{2}, \ldots, b_{n}\right\}$. Hence, we conclude that function $V(t, \mathbf{v}, i)$ is a Lyapunov-B function for $(8)$. Let $C=\max$ $\left\{c_{1}, c_{2}, \ldots, c_{n}\right\}$, it follows easily that

$$
\int_{G} V(t, \mathbf{v}, i) d \mathbf{x}=\sum_{k=1}^{n} c_{k} \int_{G} V_{k}\left(t, \mathbf{v}_{k}, i\right) d \mathbf{x} \leq \sum_{k=1}^{n} C \mu_{2}\left(\left\|\mathbf{v}_{k}\right\|\right) \leq n C \mu_{2}(\|\mathbf{v}\|)
$$

where $\|\mathbf{v}\|=\sum_{i=1}^{n}\left\|\mathbf{v}_{k}\right\|$, obviously, $\|\mathbf{v}\| \geq\left\|\mathbf{v}_{k}\right\|$. By Theorem 4.2 .3 in (Mao (1997) [39]), the trivial solution of (8) is stochastically asymptotically stable. Furthermore, making use of Condition B4 yields

$$
\lim _{\|\mathbf{v}\| \rightarrow \infty} \inf _{t \geq 0} \int_{G} V(t, \mathbf{v}, i) d \mathbf{x}=\lim _{\left\|\mathbf{v}_{k}\right\| \rightarrow \infty} \inf _{t \geq 0}\left(\sum_{i=1}^{n} c_{k} \int_{G} V_{k}\left(t, \mathbf{v}_{k}, i\right) d \mathbf{x}\right)=\infty .
$$

Then, the trivial solution of (8) is globally asymptotically stable in probability. The proof is complete.

Theorem 3. Suppose that the following conditions hold.

C1. Assume that there exist positive functions $V_{k}\left(t, \mathbf{v}_{k}, i\right) \in C^{1,2}\left(\mathbb{R}_{+} \times \mathbb{R}^{n_{k}} \times \mathbb{S} ; \mathbb{R}_{+}\right)$, and constants $p>0, q_{1}>0, q_{2} \geq 0$ satisfying

(I) $V_{k}(t, \mathbf{v}, i)$ is separated as to variables $\mathbf{v}_{k}(k \in N)$ for $i \in \mathbb{S}$;

(II)

$$
q_{1}\left\|\mathbf{v}_{k}\right\|_{G}^{p} \leq\left|\int_{G} V_{k}\left(t, \mathbf{v}_{k}, i\right) d \mathbf{x}\right|
$$

hold for $\forall\left(t, \mathbf{v}_{k}(t, \mathbf{x})\right) \in\left[t_{0}, \infty\right) \times s_{h}^{n_{k}}$, where $\mathbf{v}_{k}(t, \cdot) \in \mathbb{s}_{h}^{n_{k}}=\left\{\zeta: G \rightarrow \mathbb{R}^{n_{k}} \| \int_{G} \zeta(\mathbf{x}) d \mathbf{x} \mid<h\right\} ;$

(III) $\left\|\left(\frac{\partial V_{k}\left(t, \mathbf{v}_{k}, i\right)}{\partial \mathbf{v}_{k}}\right)^{T}\left(g_{k}\left(t, \mathbf{x}, \mathbf{v}_{k}\left(t, \mathbf{x}, t_{0}, \mathbf{v}_{k 0}\right), i\right)+\sum_{j=1}^{N} N_{k j}\left(\mathbf{v}_{k}, \mathbf{v}_{j}, t, i\right)\right)\right\|_{G}^{2} \geq q_{2}\left\|V_{k}\left(t, \mathbf{v}_{k}, i\right)\right\|_{G}^{2}$, for any $\mathbf{v}_{k} \neq 0, k \in$ $N,\left(t, \mathbf{x}_{k}\right) \in \mathbb{R}_{+} \times G$ for $i \in \mathbb{S}$.

C2. There exist functions $F_{k j}\left(\mathbf{v}, \mathbf{v}_{j}, t\right)$ and constants $a_{k j} \geq 0, b_{k}>0$ such that

$$
\mathcal{L} \int_{G} V_{k}\left(t, \mathbf{v}_{k}, i\right) d \mathbf{x} \leq-b_{k} \int_{G} V_{k}\left(t, \mathbf{v}_{k}, i\right) d \mathbf{x}+\sum_{j=1}^{n} a_{k j} F_{k j}\left(\mathbf{v}_{k}, \mathbf{v}_{j}, t\right),
$$

$t \geq t_{0}$, for $k=1,2, \ldots, N . i \in \mathbb{S}$. Here $L V_{k}$ is defined in (9).

C3. Condition A2 holds, or if $(g, A)$ is balanced and (24) holds.

Then

$$
\lim _{t \rightarrow \infty} \sup \frac{1}{t} \lg \left\|\mathbf{v}\left(t, \mathbf{x}, t_{0}, \mathbf{v}_{0}, i\right)\right\|_{G} \leq \frac{-2 \sum_{k=1}^{N} c_{k} b_{k}-q_{2}}{2 p} \text { a.s. }
$$

holds. The function $V(t, \mathbf{v}, i) \triangleq \sum_{i=1}^{n} c_{k} V_{k}\left(t, \mathbf{v}_{k}, i\right)$ is a Lyapunov-B function for (8), in which $c_{k}$ is defined as (5). Particularly, the trivial solution of (8) is almost surely exponentially stable.

Proof. For any $\mathbf{v}_{k 0} \neq 0$, denote $\mathbf{v}_{k}(t, \mathbf{x}) \triangleq \mathbf{v}_{k}\left(t, \mathbf{x}, t_{0}, \mathbf{v}_{k 0}\right)$. It follows from Lemma 3 that $\mathbf{v}_{k}(t, \mathbf{x}) \neq 0$ holds almost surely for all, $(t, \mathbf{x}) \in\left(t_{0}, \infty\right) \times G, i \in \mathbb{S}$. Applying the Itô formula to (8) gives

$$
\begin{aligned}
& d\left(\int_{G} V_{k}\left(t, \mathbf{v}_{k}(t, \mathbf{x}), \gamma(t)\right) d \mathbf{x}\right)=\left[\int_{G} \mathcal{L} V_{k}\left(t, \mathbf{v}_{k}(t, \mathbf{x}), i\right) d \mathbf{x}+\int_{G}\left(\frac{\partial V_{k}\left(t, \mathbf{v}_{k}, i\right)}{\partial \mathbf{v}_{k}}\right)^{T} \Delta \mathbf{v}_{k}(t, \mathbf{x}) d \mathbf{x}\right] d t \\
& \quad+\int_{G}\left(\frac{\partial V_{k}\left(t, \mathbf{v}_{k}, i\right)}{\partial \mathbf{v}_{k}}\right)^{T}\left[g_{k}\left(t, \mathbf{x}, \mathbf{v}_{k}(t, \mathbf{x}), i\right)+\sum_{j=1}^{N} N_{k j}\left(\mathbf{v}_{k}, \mathbf{v}_{j}, t, i\right)\right] d \mathbf{x} d W(t) .
\end{aligned}
$$

By condition C1(I), we have $\frac{\partial^{2} V_{k}\left(t, \mathbf{v}_{k}\right)}{\partial \mathbf{v}_{k} \partial \mathbf{v}_{j}}=0,(k \neq j, i, j \in N)$. From integration by parts and condition C1. (III) together with boundary condition, we obtain

$$
\begin{aligned}
\int_{G}\left(\frac{\partial V_{k}\left(t, \mathbf{v}_{k}, i\right)}{\partial \mathbf{v}_{k}}\right)^{T} \Delta \mathbf{v}_{k}(t, \mathbf{x}) d \mathbf{x}= & \left(\sum_{m=1}^{n_{k}} \sum_{j=1}^{r} \frac{\partial V_{k}}{\partial v_{k m}} D_{m j}(t, \mathbf{x}, \mathbf{v}) \frac{\partial v_{k m}}{\partial x_{j}}\right)_{\partial G} \\
& -\int_{G} \sum_{m=1}^{n_{k}} \sum_{j=1}^{r} D_{m j}(t, \mathbf{x}, \mathbf{v}) \frac{\partial^{2} V_{k}}{\partial v_{k m}^{2}}\left(\frac{\partial v_{k m}}{\partial x_{j}}\right)^{2} d \mathbf{x} \leq 0
\end{aligned}
$$


where $\mathbf{v}_{k}=\left(v_{k 1}, \ldots, v_{k n_{k}}\right)^{T}$. By conditions $\mathrm{C} 2$ and C3, we can show in the same way as in the proof of Theorem 1 that

$$
\mathscr{L} \int_{G} V(t, \mathbf{v}, i) d \mathbf{x}=\sum_{i=1}^{n} c_{k} \mathcal{L} \int_{G} V_{k}\left(t, \mathbf{v}_{k}, i\right) d \mathbf{x} \leq-\sum_{i=1}^{n} c_{k} b_{k} \int_{G} V_{k}\left(t, \mathbf{v}_{k}, i\right) d \mathbf{x} \leq-b \int_{G} V(t, \mathbf{v}, i) d \mathbf{x},
$$

where $b=\min \left\{b_{1}, b_{2}, \ldots, b_{n}\right\}$. Hence, we conclude that function $V(t, \mathbf{v}, i)$ is a Lyapunov-B function for (8). From (32)-(34), we get

$$
\begin{gathered}
\log \left(\int_{G} V_{k}\left(t, \mathbf{v}_{k}(t, \mathbf{x}), i\right) d \mathbf{x}\right) \leq \log \left(\int_{G} V_{k}\left(t_{0}, \mathbf{v}_{0}, \gamma_{0}\right) d \mathbf{x}\right)-\sum_{i=1}^{n} c_{k} b_{k}\left(t-t_{0}\right)+M(t) \\
-\frac{1}{2} \int_{t_{0}}^{t} \frac{\left|\int_{G}\left(\frac{\partial V_{k}\left(s, \mathbf{v}_{k}, i\right)}{\partial \mathbf{v}_{k}}\right)^{T}\left[g_{k}\left(s, \mathbf{x}, \mathbf{v}_{k}(s, \mathbf{x}), i\right)+\sum_{j=1}^{N} N_{k j}\left(\mathbf{v}_{k}, \mathbf{v}_{j}, s, i\right)\right] d \mathbf{x}\right|^{2}}{\left(\int_{G} V_{k}\left(s, \mathbf{v}_{k}(s, \mathbf{x}), i\right) d \mathbf{x}\right)^{2}} d s
\end{gathered}
$$

where

$$
M(t)=\int_{t_{0}}^{t} \frac{\int_{G}\left(\frac{\partial V_{k}\left(s, \mathbf{v}_{k}, i\right)}{\partial \mathbf{v}_{k}}\right)^{T}\left[g_{k}\left(s, \mathbf{x}, \mathbf{v}_{k}(s, \mathbf{x}), i\right)+\sum_{j=1}^{N} N_{k j}\left(\mathbf{v}_{k}, \mathbf{v}_{j}, s, i\right)\right] d \mathbf{x}}{\int_{G} V_{k}\left(s, \mathbf{v}_{k}(s, \mathbf{x}), i\right) d \mathbf{x}} d W(s) .
$$

It is obvious that $M(t)$ is a continuous martingale with initial value $M\left(t_{0}\right)=0$ when $i=\gamma_{0}$. Assign $\varepsilon \in(0,1)$ arbitrarily and let $n=1,2, \ldots$. It can be deduced from the exponential martingale inequality that

$$
\begin{aligned}
& \mathbb{P}\left\{\sup _{t_{0} \leq t \leq t_{0}+n}\left[M(t)-\frac{\varepsilon}{2} \int_{t_{0}}^{t} \frac{\left|\int_{G}\left(\frac{\partial V_{k}\left(s, \mathbf{v}_{k}, i\right)}{\partial \mathbf{v}_{k}}\right)^{T}\left[g_{k}\left(s, \mathbf{x}, \mathbf{v}_{k}(s, \mathbf{x}), i\right)+\sum_{j=1}^{N} N_{k j}\left(\mathbf{v}_{k}, \mathbf{v}_{j}, s, i\right)\right] d \mathbf{x}\right|^{2}}{\left(\int_{G} V_{k}\left(s, \mathbf{v}_{k}(s, \mathbf{x}), i\right) d \mathbf{x}\right)^{2}} d s\right]>\frac{2}{\varepsilon} \log n\right\} \\
& \leq \frac{1}{n^{2}} .
\end{aligned}
$$

From Lemma Borel-Cantelli [40], it is easy to see there is a corresponding integer $n_{0}=n_{0}(\omega)$ such that if $n \geq n_{0}$

$$
M(t) \leq \frac{\varepsilon}{2} \int_{t_{0}}^{t} \frac{\left|\int_{G}\left(\frac{\partial V_{k}\left(s, \mathbf{v}_{k}, i\right)}{\partial \mathbf{v}_{k}}\right)^{T}\left[g_{k}\left(s, \mathbf{x}, \mathbf{v}_{k}(s, \mathbf{x}), i\right)+\sum_{j=1}^{N} N_{k j}\left(\mathbf{v}_{k}, \mathbf{v}_{j}, s, i\right)\right] d \mathbf{x}\right|^{2}}{\left(\int_{G} V_{k}\left(s, \mathbf{v}_{k}(s, \mathbf{x}), i\right) d \mathbf{x}\right)^{2}} d s+\frac{2}{\varepsilon} \log n
$$

holds for all $t_{0} \leq t \leq t_{0}+n$. Substituting the above inequality to (35) and noting condition C1(III), we obtain that

$$
\log \left(\int_{G} V_{k}\left(t, \mathbf{v}_{k}(t, \mathbf{x}), i\right) d \mathbf{x}\right) \leq \log \left(\int_{G} V_{k}\left(t_{0}, \mathbf{v}_{0}, \gamma_{0}\right) d \mathbf{x}\right)-\frac{(1-\varepsilon) q_{2}+2 \sum_{i=1}^{n} c_{k} b_{k}}{2}\left(t-t_{0}\right)+\frac{2}{\varepsilon} \log n
$$

holds for all $t_{0} \leq t \leq t_{0}+n$ and $n \geq n_{0}$ almost surely. Thus, for almost all $\omega \in \Omega$, when $t_{0}+n-1 \leq t \leq t_{0}+n, n \geq n_{0}$

$$
\begin{aligned}
\frac{1}{t} \log \left(\int_{G} V_{k}\left(t, \mathbf{v}_{k}(t, \mathbf{x}), i\right) d \mathbf{x}\right) \leq & -\frac{\left(t-t_{0}\right)}{2 t}\left[(1-\varepsilon) q_{2}+2 \sum_{i=1}^{n} c_{k} b_{k}\right] \\
& +\frac{\log \left(\int_{G} V_{k}\left(t_{0}, \mathbf{v}_{0}, \gamma_{0}\right) d \mathbf{x}\right)+\frac{2}{\varepsilon} \log n}{t_{0}+n-1} \text { a.s. }
\end{aligned}
$$

This implies

$$
\lim _{t \rightarrow \infty} \sup \frac{1}{t} \log \left(\int_{G} V_{k}\left(t, \mathbf{v}_{k}(t, \mathbf{x}), i\right) d \mathbf{x}\right) \leq-\frac{(1-\varepsilon) q_{2}+2 \sum_{i=1}^{n} c_{k} b_{k}}{2} \text { a.s. }
$$


Combining condition $\mathrm{C} 1(\mathrm{I})$, we infer

$$
\lim _{t \rightarrow \infty} \sup \frac{1}{t} \log \left\|V_{k}\left(t, \mathbf{v}_{k}(t, \mathbf{x}), i\right)\right\| \leq-\frac{(1-\varepsilon) q_{2}+2 \sum_{i=1}^{n} c_{k} b_{k}}{2 p} \text { a.s. }
$$

and the required assertion (31) follows since $\varepsilon>0$ is arbitrary. The proof is complete.

Remark 2. If in $C 2, b_{k}>0$ is changed into $b_{k} \in \mathbb{R}$ such that $2 \sum_{k=1}^{N} c_{k} b_{k}<q_{2}$, obviously, the trivial solution of (8) is almost surely exponentially stable.

Corollary 2. Suppose that the following conditions hold.

D1. Assume that there exist positive functions $V_{k}\left(t, \mathbf{v}_{k}, i\right) \in C^{1,2}\left(\mathbb{R}_{+} \times \mathbb{R}^{n_{k}} \times \mathbb{S} ; \mathbb{R}_{+}\right)$, and constants $p>0, \alpha>0$ satisfying

(I) $V_{k}(t, \mathbf{v}, i)$ is separated as to variables $\mathbf{v}_{k}(k \in N)$ for $i \in \mathbb{S}$;

(II)

$$
\alpha\left\|V_{k}\left(t, \mathbf{v}_{k}, i\right)\right\|_{G}^{p} \leq \int_{G} V_{k}\left(t, \mathbf{v}_{k}, i\right) d \mathbf{x}
$$

$$
\text { hold for } \forall\left(t, \mathbf{v}_{k}(t, \mathbf{x})\right) \in\left[t_{0}, \infty\right) \times \mathbb{s}_{h}^{n_{k}} \text {, where } \mathbf{v}_{k}(t, \cdot) \in s_{h}^{n_{k}}=\left\{\zeta: G \rightarrow \mathbb{R}^{n_{k}} \| \int_{G} \zeta(\mathbf{x}) d \mathbf{x} \mid<h\right\} \text {; }
$$

(III) $\left\|\left(\frac{\partial V_{k}\left(t, \mathbf{v}_{k}, i\right)}{\partial \mathbf{v}_{k}}\right)^{T}\left(g_{k}\left(t, \mathbf{x}, \mathbf{v}_{k}\left(t, \mathbf{x}, t_{0}, \mathbf{v}_{k 0}\right), i\right)+\sum_{j=1}^{N} N_{k j}\left(\mathbf{v}_{k}, \mathbf{v}_{j}, t, i\right)\right)\right\|_{G}^{2} \geq q_{2}\left\|V_{k}\left(t, \mathbf{v}_{k}, i\right)\right\|_{G}^{2}$, for any $\mathbf{v}_{k} \neq 0, k \in$ $N,\left(t, \mathbf{x}_{k}\right) \in \mathbb{R}_{+} \times G$ for $i \in \mathbb{S}$.

D2. There exist functions $F_{k j}\left(\mathbf{v}, \mathbf{v}_{j}, t\right)$ and constants $a_{k j} \geq 0, \lambda>0$ such that

$$
\mathcal{L} \int_{G} V_{k}\left(t, \mathbf{v}_{k}, i\right) d \mathbf{x} \leq-\lambda \int_{G} V_{k}\left(t, \mathbf{v}_{k}, i\right) d \mathbf{x}+\sum_{j=1}^{n} a_{k j} F_{k j}\left(\mathbf{v}_{k}, \mathbf{v}_{j}, t\right),
$$

$t \geq t_{0}$, for $k=1,2, \ldots, N$. $i \in \mathbb{S}$. Here $L V_{k}$ is defined in (9).

D3. Condition A2 holds, or if $(g, A)$ is balanced and (24) holds.

Then

$$
\lim _{t \rightarrow \infty} \sup \frac{1}{t} \lg \left\|\mathbf{v}\left(t, \mathbf{x}, t_{0}, \mathbf{v}_{0}, i\right)\right\|_{G} \leq \frac{-\lambda \sum_{k=1}^{N} c_{k}}{p} \text { a.s. }
$$

holds. The function $V(t, \mathbf{v}, i) \triangleq \sum_{i=1}^{n} c_{k} V_{k}\left(t, \mathbf{v}_{k}, i\right)$ is a Lyapunov-B function for (8), in which $c_{k}$ is defined as (5). Particularly, the trivial solution of $(8)$ is almost surely exponentially stable.

Proof. This corollary is the direct result of Theorem 3, if we choose $q_{1}=\alpha, b_{k}=\lambda$ and $q_{2}=0$.

Remark 3. In this paper, the novel methods of constructing a Lyapunov function to study stability conditions of the SCRDSNs are proposed. Ours are different from some other latest methods on constructing the Lyapunov function [41-46], because our methods build a relation between the stability criteria of a CSN and some topology property of the network. Topology property of the networks is of great importance to the dynamical analysis for the coupled networks. Therefore, for dealing with CSN, our methods are less conservative.

\section{Example}

Consider the 2-dimensional Itô SRDSMS (1) satisfying the bounded condition (2), and we assume $(q, A)$ is strongly connected and balanced. The Markov chain $\gamma(\cdot)$ is independent of the Brownian motion $W(\cdot)$.

$$
\left\{\begin{aligned}
d v_{1}(t, x)= & {\left[\Delta v_{1}(t, x)+\alpha(\gamma(t)) v_{2}(t, x)-\alpha(\gamma(t)) v_{1}(t, x)-\alpha(\gamma(t)) \sum_{j=1}^{2} a_{1 j}\left(v_{1}(t, x)-v_{j}(t, x)\right)\right] d t, } \\
d v_{2}(t, x)= & {\left[\Delta v_{2}(t, x)-\alpha(\gamma(t)) v_{1}(t, x)-2 \alpha(\gamma(t)) v_{2}(t, x)+\alpha(\gamma(t)) \sum_{j=1}^{2} a_{2 j}\left(v_{2}(t, x)-v_{j}(t, x)\right)\right] d t } \\
& +\sqrt{\alpha(\gamma(t))} v_{2}(t, x) d w(t) .
\end{aligned}\right.
$$

Construct function $V=\left(\int_{G} v_{1}(t, x) d x\right)^{2}+\left(\int_{G} v_{2}(t, x) d x\right)^{2}$, we have

$$
\int_{G} v(t, x) d x \geq \frac{1}{\|G\|}\|v\| \text {. }
$$


Besides,

$$
\begin{aligned}
& \mathcal{L} \int_{G} V d x=\int_{G} \mathcal{L} V d x=\alpha(\gamma(t)) \int_{G}\left[2 v_{1} v_{2}-2 v_{1}^{2}-2 v_{1} v_{2}-4 v_{2}^{2}+v_{2}^{2}\right] d x+\sum_{j=1}^{2} a_{k j} F_{k j}\left(v_{k}, v_{j}\right) \\
& =-2 \alpha(\gamma(t)) \int_{G} V d x<0
\end{aligned}
$$

where $F_{k j}\left(v_{k}, v_{j}\right)=2 \int_{G} v_{k}^{2}-v_{j}^{2} d x$. It is easy to see that along every directed cycle $\mathrm{C}$ of the weighted digraph $(g, A)$,

$$
\sum_{(k, j) \in E\left(C_{Q}\right)}\left[F_{j k}\left(\mathbf{v}_{j}, \mathbf{v}_{k}, t\right)+F_{k j}\left(\mathbf{v}_{k}, \mathbf{v}_{j}, t\right)\right]=0 .
$$

According to Corollary 1, we deduce

$$
\lim _{t \rightarrow \infty} \sup \frac{1}{t} \lg \left\|\mathbf{v}\left(t, \mathbf{x}, t_{0}, \mathbf{v}_{0}, i\right)\right\|_{G} \leq-2 \text { a.s. }
$$

Therefore, the trial solution of system (43) is almost surely exponentially stable.

\section{Conclusions}

In this paper, we have investigated some stabilities of some stochastic coupled reaction-diffusion systems on networks (MSRDCSNs). First, a MSRDCSNs model has been proposed. Then, a systematic method for constructing a global Lyapunov function for MSRDCSNs by using graph theory has been presented. This method overcomes the difficulty in finding Lyapunov function for a coupled system. At last, some sufficient conditions for stability of MSRDCSNs are obtained. Future work is to give a systematic approach to build a Lyapunov function for coupled impulsive reaction-diffusion systems on networks.

\section{Acknowledgments}

The authors would like to thank the editors and the anonymous reviewers for their valuable comments and constructive suggestions. This research is supported by the Natural Science Foundation of Guangxi Autonomous Region (No. 2012GXNSFBA053003, 2013YB141), the National Natural Science Foundations of China $(60973048,61272077,60974025$, 60673101, 60939003), National 863 Plan Project (2008 AA04Z401, 2009AA043404), the Natural Science Foundation of Shandong Province (No. Y2007G30), the Scientific and Technological Project of Shandong Province (No. 2007GG3WZ04016), the Science Foundation of Harbin Institute of Technology (Weihai) (HIT(WH) 200807), the Natural Scientific Research Innovation Foundation in Harbin Institute of Technology (HIT.NSRIF. 2001120), the China Postdoctoral Science Foundation (2010048 1000) and the Shandong Provincial Key Laboratory of Industrial Control Technique (Qingdao University).

\section{References}

[1] S. Strogatz, Exploring complex networks, Nature 410 (8) (2011) 268-276.

[2] D. Watts, S. Strogatz, Collective dynamics of small-world networks, Nature 393 (6684) (1997) 440-442.

[3] P. Satorras, A. Vespignani, Epidemic dynamics and endemic states in complex networks, Phys. Rev. E 63 (2001) 066117.

[4] S. Boccaletti, V. Latora, Y. Moreno, M. Chavez, D.U. Hwang, Complex networks: structure and dynamics, Phys. Rep. 424 (2006) 175-308.

[5] A.E. Motter, C. Zhou, J. Kurths, Network synchronization, diffusion, and the paradox of heterogeneity, Phys. Rev. E 71 (2005) 016116.

[6] T. Nishikawa, A. Motter, E. Lai, Y. Hoppensteadt, Heterogeneity in oscillator networks: are smaller worlds easier to synchronize? Phys. Rev. Lett. 91 (1) (2003) 014101.

[7] C.W. Wu, On the relationship between pinning control effectiveness and graph topology in complex networks of dynamical systems, Chaos 18 (2008) 037103.

[8] M.Y. Li, Z. Shuai, Global-stability problem for coupled systems of differential equations on networks, J. Differential Equations 248 (2010) 1-20.

[9] J. Lu, D.W.C. Ho, J. Cao, J. Kurths, Exponential synchronization of linearly coupled neural networks with impulsive disturbances, IEEE Trans. Neural Netw. 22 (2) (2011) 329-335.

[10] J. Lu, D.W.C. Ho, J. Cao, A unified synchronization criterion for impulsive dynamical networks, Automatica 46 (2010) 1215-1221.

[11] W. Yu, J. Cao, W. Lu, Synchronization control of switched linearly coupled neural networks with delay, Neurocomputing 73 (2010) 858-866

[12] Y.G Kao, H. Sun, H.X Cao, Stability analysis for coupled stochastic systems with time delay on networks, Math. Appl. 26 (2013) 67-75.

[13] X.X Liao, S.Z. Yang, S.J. Cheng, Y.L. Fu, Stability of general neural networks with reaction-diffusion, Sci. China F. 44 (5) (2001) 389-395.

[14] X.X Liao, Theory and Application of Stability for Dynamical Systems, Defense Industry Press, Beijing, 2000.

[15] L.S. Wang, D.Y. Xu, Global exponential stability of variable delay reaction diffusion Hopfield neural networks, Sci. China F. 46 (6) (2003) $466-474$.

[16] Q. Luo, Y.Zhang, Almost sure exponential stability of stochastic reaction diffusion systems, Nonlinear Anal. TMA 71 (12) (2009) e487-e493.

[17] Q. Luo, F.Q. Deng, J.D. Bao, B.R. Zhao, Y.L. Fu, Stabilization of stochastic Hopfield neural network with distributed parameters, Sci. China F 47 (6) (2004) $752-762$.

[18] C.H Wang, Y.G. Kao, G. Yang, Exponential stability of impulsive stochastic fuzzy reaction-diffusion Cohen-Grossberg neural networks with mixed delays, Neurocomputing 89 (2012) 55-63.

[19] Y.G. Kao, C.H. Wang, Global stability analysis for stochastic coupled reaction-diffusion systems on networks, Nonlinear Anal. Ser. B. RWA 14 (3) (2013) $1457-1465$.

[20] N.N. Krasovskii, E.A. Lidskii, Analysis and design of controllers in systems with random attributes, Autom. Remote Control 22 (1961) 1021-1025

[21] Yan Liu, Stochastic asymptotic stability of Markovian jumping neural networks with Markov mode estimation and mode-dependent delays, Phys. Lett. A 373 (41) (2009) 3741-3742.

[22] X. Mao, C. Yuan, Stochastic Differential Equations with Markovian Switching, Imperial College Press, 2006. 
[23] Y. Kao, J. Guo, C. Wang, X. Sun, Delay-dependent robust exponential stability of Markovian jumping reaction-diffusion Cohen-Grossberg neural networks with mixed delays, J. Franklin Inst. 349 (6) (2012) 1972-1988.

[24] Lixian Zhang, E. Boukas, Stability and stabilization of Markovian jump linear systems with partly unknown transition probability, Automatica 45 (2) (2009) 463-468

[25] Lixian Zhang, J. Lam, Necessary and sufficient conditions for analysis and synthesis of Markov jump linear systems with incomplete transition descriptions, IEEE Trans. Automat. Control 55 (7) (2010) 1695-1701.

[26] Lixian Zhang, E. Boukas, Mode-dependent $H_{\infty}$ filtering for discrete-time Markovian jump linear systems with partly unknown transition probability, Automatica 45 (6) (2009) 1462-1467.

[27] Lixian Zhang, $H_{\infty}$ estimation for piecewise homogeneous Markov jump linear systems, Automatica 45 (11) (2009) $2570-2576$.

[28] Lixian Zhang, E. Boukas, J. Lam, Analysis and synthesis of Markov jump linear systems with time-varying delays and partially known transition probabilities, IEEE Trans. Automat. Control 53 (10) (2008) 2458-2464.

[29] Z. Wu, P. Shi, H. Su, $\mathrm{H}_{2} \mathrm{H}_{\infty}$ filter design for discrete-time singular Markovian jump systems with time-varying delays, Inform. Sci. 181 (24) (2011) 5534-5547.

[30] Fei Zhongyang, Gao Huijun, Shi Peng, New results on stabilization of Markovian jump systems with time delay, Automatica 45 (10) (2009) $2300-2306$.

[31] Ou Yan, Shi Peng, Liu Hongyang, A mode-dependent stability criterion for delayed discrete-time stochastic neural networks with Markovian jumping parameters, Neurocomputing 73 (7-9) (2010) 1491-1500.

[32] Liu Ming, Shi Peng, Zhang Lixian, Fault-tolerant control for nonlinear Markovian jump systems via proportional and derivative sliding mode observer technique, IEEE Trans. Circuits Sys. I 11 (2011) 2755-2764.

[33] Wu Ligang, Shi Peng, Gao Huijun, H-infinity filtering for 2D Markovian jump systems, Automatica 44 (7) (2008) 1849-1858.

[34] G. Yin, F. Xi, Stability of regime-switching jump diffusions, SIAM J. Control Optim. 48 (2010) 4525-4549.

[35] G. Yin, C. Zhu, Properties of solutions of stochastic differential equations with continuous-state-dependent switching, J. Differential Equations 249 (2010) 2409-2439.

[36] G. Yin, Y. Sun, L.Y. Wang, Asymptotic properties of consensus-type algorithms for networked systems with regime-switching topologies, Automatica 47 (2011) 1366-1378.

[37] Z. Yang, G. Yin, Stability of nonlinear regime-switching jump diffusions, Nonlinear Anal. Ser. A TMA 75 (2012) $3854-3873$.

[38] F. Wu, G. Yin, L.Y. Wang, Moment exponential stability of random delay systems with two-time-scale Markovian switching, Nonlinear Anal. Ser. B RWA 13 (2012) 2476-2490.

[39] X. Mao, Stochastic Differential Equations and Their Applications, Horwood Publishing, Chichester, 1997.

[40] X.R. Mao, Exponential Stability of Stochastic Differential Equations, Marcel Dekker, New York, 1994.

[41] Rongni Yang, Huijun Gao, Peng Shi, Novel robust stability criteria for stochastic Hopfield neural networks with time delays, IEEE Trans. Syst. Man Cybern. B 39 (2) (2009) 467-474.

[42] Rongni Yang, Zexu Zhang, Peng Shi, Exponential stability on stochastic neural networks with discrete interval and distributed delays, IEEE Trans. Neural Netw. 21 (1) (2010) 169-175.

[43] Ligang Wu, Xiaojie Su, Peng Shi, Jianbin Qiu, A new approach to stability analysis and stabilization of discrete-time fuzzy time-varying delay systems, IEEE Trans. Syst. Man Cybern. B 41 (1) (2011) 273-286.

[44] Ligang Wu, Xiaojie Su, Peng Shi, Jianbin Qiu, Model approximation for discrete-time state-delay systems in the T-S fuzzy framework, IEEE Trans. Fuzzy Syst. 19 (2) (2011) 366-378.

[45] X. Su, P. Shi, L. Wu, Y. Song, A novel approach to filter design for T-S fuzzy discrete-time systems with time-varying delay, IEEE Trans. Fuzzy Syst. 20 (6) (2012) 1114-1129.

[46] P. Balasubramaniam, C. Vidhya, Exponential stability of stochastic reaction-diffusion uncertain fuzzy neural networks with mixed delays and Markovian jumping parameters, Expert Syst. Appl. 39 (2012) 3109-3115. 